\title{
On Generalized Lipschitz Classes and Fourier Series
}

\author{
Sergey Tikhonov
}

\begin{abstract}
In 1967 R.P. Boas Jr. found necessary and sufficient conditions of belonging of a function to a Lipschitz class. Later Boas's findings were generalized by many authors (M. and S. Izumi (1969), L.-Y. Chan (1991) and others). Recently, L. Leindler (2000) and J. Nemeth (2001) have published two papers, in which they have generalized all the previous results. The authors have considered the case, when the order of modulus of smoothness equals one (L. Leindler) or two (J. Nemeth). In this paper, we prove theorems of Boas-type for the modulus of smoothness of any order. Furthermore, we solve the inverse problem. Also, we discuss some conditions on a majorant which are equivalent to the well-known conditions of Bari-Stechkin.
\end{abstract}

Keywords: Lipschitz class, trigonometric series, moduli of smoothness of fractional order

MSC 2000: 26A16, 42A16, 40A05, 26A 15

\section{Introduction}

In 1967, R.P. Boas Jr. [4] proved a series of theorems on the connection between the behaviour of Fourier coefficients of a function $f$ and its structural properties described by the modulus of continuity. Namely, he investigated the function class Lip $\alpha(0<\alpha \leq 1)$ from this point of view. In 1969, M. and S. Izumi [6] generalized his results. They introduced the following function classes. Let $\varphi(t)$ be a positive and nondecreasing function defined on the interval $(0,1)$. The $\operatorname{Lip} \varphi(t)$ and $\Lambda(\varphi(t))$ classes are defined as follows:

$$
\begin{aligned}
& \operatorname{Lip} \varphi(t):=\left\{f: \sup _{t, x}\left(\frac{|f(x+t)-f(x)|}{\varphi(t)}\right)<\infty\right\} \\
& \Lambda(\varphi(t)):=\left\{f: \sup _{t, x}\left(\frac{|f(x+t)-2 f(x)+f(x-t)|}{\varphi(t)}\right)<\infty\right\} .
\end{aligned}
$$

S. Tikhonov: Moscow State University, Moscow, RUSSIA; present address: Centre de Recerca Matematica (CRM), Bellaterra (Barcelona), SPAIN; tikhonov@mccme.ru 
Theorem 1.1. ([6]) Let $\lambda_{n} \geq 0$ be the Fourier sine or cosine coefficients of $\psi(x)$. Let $\varphi(t)$ be a positive and nondecreasing function on $(0,1)$, satisfying the condition

$$
\int_{0}^{t} \varphi(u) u^{-1} d u \leq K \varphi(t) \quad \text { as } \quad t \rightarrow 0
$$

and

$$
\int_{t}^{1} \varphi(u) u^{-2} d u \leq K \varphi(t) t^{-1} \quad\left(\text { or } \int_{t}^{1} \varphi(u) u^{-3} d u \leq K \varphi(t) t^{-2}\right) \text { as } t \rightarrow 0 .
$$

Then $\psi \in \operatorname{Lip} \varphi(t)($ or $\psi \in \Lambda(\varphi(t)))$ if and only if

$$
\sum_{k=n / 2}^{n} \lambda_{k} \leq K \varphi\left(\frac{1}{n}\right) \quad \text { as } \quad n \rightarrow \infty
$$

or, equivalently,

$$
\sum_{k=n}^{\infty} \lambda_{k} \leq K \varphi\left(\frac{1}{n}\right) \quad \text { as } \quad n \rightarrow \infty .
$$

In 1990, Boas's results were also generalized by J. Nemeth [12] who used the so-called generalized Lipschitz and Zigmund classes and replaced the function $t^{\alpha}$ by a more general function $\omega_{\alpha}(t)$.

Let $^{1} \Omega_{\alpha}(0 \leq \alpha \leq 1)$ denote the set of nonnegative nondecreasing functions $\omega(\delta)=\omega_{\alpha}(\delta)$ on $[0,1]$, which have the following properties:

$\left(\Omega_{\alpha}^{(\mathbf{1})}\right)$ for any $\alpha^{\prime}>\alpha$ there exists a natural number $\mu=\mu\left(\alpha^{\prime}\right)$ such that

$$
2^{\mu \alpha^{\prime}} \omega_{\alpha}\left(2^{-n-\mu}\right)>2 \omega_{\alpha}\left(2^{-n}\right) \quad \text { holds for all } n(\geq 1)
$$

$\left(\Omega_{\alpha}^{(2)}\right)$ for every natural $\nu$ there exists a natural number $N:=N(\nu)$ such that

$$
2^{\nu \alpha} \omega_{\alpha}\left(2^{-n-\nu}\right) \leq 2 \omega_{\alpha}\left(2^{-n}\right) \quad \text { if } \quad n>N
$$

Theorem 1.2. ([12]) Let $0<\alpha<1$. Let $\omega_{\alpha} \in \Omega_{\alpha}$ be the modulus of continuity, and, $\lambda_{n} \geq 0$ be the Fourier sine or cosine coefficients of $\psi(x)$. Then $\omega_{1}(\psi, \delta)=$ $O\left[\omega_{\alpha}(\delta)\right]$, if and only if

$$
\sum_{k=n}^{\infty} \lambda_{k}=O\left[\omega_{\alpha}\left(\frac{1}{n}\right)\right]
$$

or, equivalently,

$$
\sum_{k=1}^{n} k \lambda_{k}=O\left[n \omega_{\alpha}\left(\frac{1}{n}\right)\right]
$$

\footnotetext{
${ }^{1}$ Note, that conditions $\left(\Omega_{\alpha}^{(1)}\right),\left(\Omega_{\alpha}^{(2)}\right)$ were introduced in [7].
} 
In the case of $\omega_{\alpha}(\delta)=\delta^{\alpha}(0<\alpha<1)$, this theorem is reduced to the results of Boas [4]. Further, L.-Y. Chan [5] proved the following Boas-type theorem.

Theorem 1.3. ([5]) Let $\varphi(\cdot)$ be the positive function such that there exist constants $0<\alpha_{1} \leq \alpha_{2}<1$ with the following conditions: $\varphi(t) t^{-\alpha_{1}}$ is a nondecreasing function and $\varphi(t) t^{-\alpha_{2}}$ is a nonincreasing function. Let $\lambda_{n} \geq 0$ be the Fourier sine or cosine coefficients of $\psi(x)$. Then $\omega_{1}(\psi, \delta)=O[\varphi(\delta)]$, if and only if

$$
\sum_{k=n}^{\infty} \lambda_{k}=O\left[\varphi\left(\frac{1}{n}\right)\right],
$$

or, equivalently,

$$
\sum_{k=1}^{n} k \lambda_{k}=O\left[n \varphi\left(\frac{1}{n}\right)\right] .
$$

Recently, L. Leindler [9] and J. Nemeth [13] have provided the generalization of all the previous results. The main concept both of them have used is the concept of quasi power-monotone sequences.

We shall say that the sequence $\gamma:=\left\{\gamma_{n}\right\}$ of positive terms is quasi monotone increasing (decreasing), if there exist a natural number $N:=N(\gamma)$ and a constant $K:=K(\gamma) \geq 1$ such that

$$
K \gamma_{n} \geq \gamma_{m} \quad\left(\gamma_{n} \leq K \gamma_{m}\right)
$$

holds for any $n \geq m \geq N$.

Theorem 1.4. ([9]) Let $\lambda_{n} \geq 0$ be the Fourier sine or cosine coefficients of $\psi(x)$. Let us assume that a given positive sequence $\left\{\gamma_{n}\right\}$ has the following property: There exists a positive $\varepsilon$, such that

$\left(\mathbf{P}_{+}\right) \quad$ the sequence $\left\{n^{\varepsilon} \gamma_{n}\right\}$ is quasi monotone decreasing

$\left(\mathbf{P}_{-}\right)$the sequence $\left\{n^{1-\varepsilon} \gamma_{n}\right\}$ is quasi monotone increasing .

Then $\omega_{1}\left(\psi, \frac{1}{n}\right)=O\left(\gamma_{n}\right)$ if and only if

$$
\sum_{k=n}^{\infty} \lambda_{k}=O\left(\gamma_{n}\right)
$$

or, equivalently,

$$
\sum_{k=1}^{n} k \lambda_{k}=O\left(n \gamma_{n}\right) .
$$

Theorem 1.5. ([13]) Let $\lambda_{n} \geq 0$ be the Fourier sine or cosine coefficients of $\psi(x)$. Let us assume that a given positive sequence $\left\{\gamma_{n}\right\}$ has the following property: There exists a positive $\varepsilon$, such that 
$\left(\mathbf{P}_{+}\right) \quad$ the sequence $\left\{n^{\varepsilon} \gamma_{n}\right\}$ is quasi monotone decreasing

$(\hat{\mathbf{P}})$ the sequence $\left\{n^{2-\varepsilon} \gamma_{n}\right\}$ is quasi monotone increasing .

Then $\omega_{2}\left(\psi, \frac{1}{n}\right)=O\left(\gamma_{n}\right)$ if and only if

$$
\sum_{k=n}^{\infty} \lambda_{k}=O\left(\gamma_{n}\right)
$$

The aim of the article is to study different ways of defining the generalized Lipschitz classes. For this purpose we shall consider conditions on a function $\varphi(t)$ in the classes $\operatorname{Lip} \varphi(t)$ and $\Lambda(\varphi(t))$ introduced by M. and S. Izumi, conditions on a function $\omega_{\alpha} \in \Omega_{\alpha}$ and conditions on a majorant given by L.-Y. Chan [5], L. Leindler [9] and J. Nemeth [13]. Further, we shall obtain the criterion of belonging of a function to the generalized Lipschitz class. Defining this class, we shall use the modulus of smoothness of positive order $\beta$ of a function $f$, and give the necessary and sufficient condition for this criterion to hold. All the results mentioned above are the particular cases of our theorems.

The outline of this paper is as follows. Section 2 introduces the basic definitions. In Section 3, we consider some useful lemmas. In Section 4, we give the interrelation between several known conditions on a majorant. Finally, in Section 5, we write the criterion of belonging of a function to the generalized Lipschitz class.

\section{Definitions}

Let $f(x)$ be a continuous function and $\|f(\cdot)\|=\max _{x \in[0,2 \pi]}|f(x)|$. The modulus of smoothness of order $\beta(\beta>0)$ of a function $f \in C$ is given by

$$
\omega_{\beta}(f, t)=\sup _{|h| \leq t}\left\|\sum_{\nu=0}^{\infty}(-1)^{\nu}\left(\begin{array}{l}
\beta \\
\nu
\end{array}\right) f(x+(\beta-\nu) h)\right\|,
$$

where $\left(\begin{array}{l}\beta \\ \nu\end{array}\right)=\frac{\beta(\beta-1) \cdots(\beta-\nu+1)}{\nu !}$ for $\nu \geq 1$ and $\left(\begin{array}{l}\beta \\ \nu\end{array}\right)=1$ for $\nu=0$. We shall say that a function $\varphi(\delta)(0<\delta \leq 1)$ is a majorant $(\varphi \in \Phi)$, if

a) $\varphi(\delta) \neq 0, \quad 0<\delta \leq 1$

b) $\varphi\left(\delta_{1}\right) \leq \varphi\left(\delta_{2}\right), \quad 0 \leq \delta_{1}<\delta_{2} \leq 1$

c) $\varphi(\delta) \rightarrow 0$ as $\delta \rightarrow 0$.

We shall consider a function $\varphi \in \Phi$ that satisfies the following conditions ${ }^{2}$ :

(B) $\quad \sum_{k=n+1}^{\infty} \frac{1}{k} \varphi\left(\frac{1}{k}\right)=O\left[\varphi\left(\frac{1}{n}\right)\right]$

\footnotetext{
${ }^{2}$ The conditions $(\mathrm{B}),\left(\mathrm{B}_{\beta}\right)$ were introduced in [2].
} 
$\left(B_{\beta}\right) \quad \sum_{k=1}^{n} k^{\beta-1} \varphi\left(\frac{1}{k}\right)=O\left[n^{\beta} \varphi\left(\frac{1}{n}\right)\right]$.

A sequence $\gamma:=\left\{\gamma_{n}\right\}$ of positive terms will be called

- almost increasing (almost decreasing), if there exists a constant $K:=$ $K(\gamma) \geq 1$ such that $(1)$ holds for any $n \geq m$,

- local almost increasing (local almost decreasing, if there exists a constant $K:=K(\gamma) \geq 1$ such that

$$
K \gamma_{n+1} \geq \gamma_{n} \quad\left(\gamma_{n+1} \leq K \gamma_{n}\right)
$$

holds for any integer $n$ (we shall write $\gamma \in L A I$ or $\gamma \in L A D$, respectively),

- lacunary starting from some member, if there exist a constant $\lambda>1$ and a natural number $N:=N(\gamma)$ such that

$$
\frac{\gamma_{n+1}}{\gamma_{n}} \geq \lambda>1 \text { holds for any } n \geq N
$$

- quasi geometrically increasing (quasi geometrically decreasing), if there exist a natural number $\mu:=\mu(\gamma), N:=N(\gamma)$ and a constant $K:=K(\gamma) \geq 1$ such that

$$
\gamma_{n+\mu} \geq 2 \gamma_{n} \text { and } \gamma_{n} \leq K \gamma_{n+1} \quad\left(\gamma_{n+\mu} \leq \frac{1}{2} \gamma_{n} \text { and } \gamma_{n+1} \leq K \gamma_{n}\right)
$$

hold for any $n \geq N$,

- bounded by blocks, if the inequalities

$$
\alpha_{1} \Gamma_{m}^{(k)} \leq \gamma_{n} \leq \alpha_{2} \Gamma_{M}^{(k)}, \quad 0<\alpha_{1} \leq \alpha_{2}<\infty
$$

hold for any $2^{k} \leq n \leq 2^{k+1}, k=1,2, \cdots$, where

$$
\Gamma_{m}^{(k)}=\min \left(\gamma_{2^{k}}, \gamma_{2^{k+1}}\right) \text { and } \Gamma_{M}^{(k)}=\max \left(\gamma_{2^{k}}, \gamma_{2^{k+1}}\right) .
$$

Also, we shall say that a sequence $\gamma:=\left\{\gamma_{n}\right\}$ of positive terms satisfies the condition $(S Q)$, if there exists $\varepsilon \in(0,1)$ such that $\left\{n^{\varepsilon} \gamma_{n}\right\}$ is almost decreasing. And $\gamma$ satisfies the condition $\left(S Q_{\beta}\right)$, if there exists $\varepsilon \in(0, \beta)$ such that $\left\{n^{\beta-\varepsilon} \gamma_{n}\right\}$ is almost increasing.

A sequence $\mathbf{c}:=\left\{c_{n}\right\}$ of positive numbers tending to zero is of rest bounded variation, or briefly $R_{0}^{+} B V S$, if it possesses the property

$$
\sum_{n=m}^{\infty}\left|c_{n}-c_{n+1}\right| \leq K(\mathbf{c}) c_{m}
$$

for all natural numbers $m$, where $K(\mathbf{c})$ is a constant depending only on $\mathbf{c}^{3}$

\footnotetext{
${ }^{3}$ This definition was introduced by L. Leindler (see [10]). It is clear that the class of monotonic sequences $M=\left\{\gamma: \gamma_{n} \downarrow 0\right\} \subset R_{0}^{+} B V S$, but the class of quasimonotonic sequences $Q M=\left\{\gamma: \exists \tau \geq 0\right.$ such that $\left.\gamma_{n} n^{-\tau} \downarrow 0\right\}$ is not comparable to the class $R_{0}^{+} B V S$ (see [10]).
} 
Now, we recall the following definition by Matuszewska (see [3, p. 68]). Let $f(\cdot)$ be positive on $(0, X], X>0$. Its upper Matuszewska index $M(f)$ is the infimum of those $\alpha$, for which there exists a constant $C=C(\alpha)$ such that for any $0<\Lambda<1$,

$$
\frac{f(\lambda x)}{f(x)} \geq C \lambda^{\alpha}\{1+o(1)\} \quad(x \rightarrow 0) \text { uniformly in } \lambda \in[\Lambda, 1]
$$

its lower Matuszewska index $m(f)$ is the supremum of those $\beta$ for which, for some $D=D(\beta)>0$ and for any $0<\Lambda<1$,

$$
\frac{f(\lambda x)}{f(x)} \leq D \lambda^{\beta}\{1+o(1)\} \quad(x \rightarrow 0) \text { uniformly in } \lambda \in[\Lambda, 1] .
$$

Let $E_{n}(f)$ be the best approximation of a function $f$ by trigonometric polynomials of order no more than $n$, i.e.

$$
E_{n}(f)=\inf _{\alpha_{k}, \beta_{k} \in \mathbb{R}}\left\|f(x)-\sum_{\nu=0}^{n}\left(\alpha_{k} \cos k x+\beta_{k} \sin k x\right)\right\| .
$$

Finally, we shall say that the continuous function $f(x)$ with Fourier series

$$
\frac{a_{0}}{2}+\sum_{n=1}^{\infty}\left(a_{n} \cos n x+b_{n} \sin n x\right)
$$

belongs to the class $C^{+}$, if the sequences of Fourier coefficients $\left\{a_{n}\right\}$ and $\left\{b_{n}\right\}$ satisfy the following condition: $a_{n} a_{m} \geq 0$ and $b_{n} b_{m} \geq 0$ holds for any integers $n$ and $m$.

\section{Auxiliary results}

We shall need the following lemmas.

Lemma 3.1. ([8]). Let $\gamma=\left\{\gamma_{n}\right\}$ be a positive sequence. Then the inequalities

$$
\sum_{k=1}^{n} \gamma_{k} \leq C \gamma_{n} \quad(n=1,2, \cdots ; C \geq 1)
$$

or

$$
\sum_{k=n}^{\infty} \gamma_{k} \leq C \gamma_{n} \quad(n=1,2, \cdots ; C \geq 1),
$$

hold if and only if the sequence $\gamma$ is quasi geometrically increasing or decreasing, respectively. 
Lemma 3.2. Let $\gamma=\left\{\gamma_{n}\right\}$ be a positive sequence. Then $\gamma$ is almost decreasing (or increasing) if and only if $\gamma$ is quasi decreasing (increasing).

Proof. It is trivial that if $\gamma$ is almost decreasing (or increasing) then $\gamma$ is quasi decreasing (increasing).

On the other hand, let $\gamma$ be a quasi decreasing sequence, i.e. there exist $N=N(\gamma) \in \mathbb{N}$ and $K_{1}=K_{1}(\gamma) \geq 1$ such that $\gamma_{n} \leq K_{1} \gamma_{m}$ holds for all $N \leq m \leq n$. Then $\gamma_{n} \leq K \gamma_{m}$ holds for any $n \geq m$ with

$$
K=K_{1} \max _{1 \leq \nu \leq N} \gamma_{\nu}\left(\min _{1 \leq \nu \leq N} \gamma_{\nu}\right)^{-1}
$$

The proof is similar for quasi increasing sequences.

By Lemma 3.2 we can write Proposition 3.3 and Lemma 4.2 from [9] in the following way (Lemmas 3.3 and 3.4).

Lemma 3.3. Let $\gamma=\left\{\gamma_{n}\right\}$ be a positive sequence bounded by blocks. Then the sequence $\left\{n^{\varepsilon} \gamma_{n}\right\}$ is almost increasing (decreasing) with a certain negative (positive) number $\varepsilon$ if and only if the sequence $\left\{\gamma_{2^{n}}\right\}$ is quasi geometrically increasing (decreasing).

Lemma 3.4. Let $\gamma=\left\{\gamma_{n}\right\}$ be a positive sequence and $\lambda=\left\{\lambda_{n}\right\}$ be a nonnegative sequence and $\delta>0$. If there exists $\varepsilon>0$ such that the sequence $\left\{n^{-\varepsilon} \gamma_{n}\right\}$ is almost increasing and the sequence $\left\{n^{\varepsilon-\delta} \gamma_{n}\right\}$ is almost decreasing, then

$$
\sum_{k=n}^{\infty} \lambda_{k}=O\left(n^{-\delta} \gamma_{n}\right)
$$

is equivalent to

$$
\sum_{k=1}^{n} k^{\delta} \lambda_{k}=O\left(\gamma_{n}\right)
$$

Lemma 3.5. Let $\gamma=\left\{\gamma_{n}\right\}$ be a positive sequence and $\varepsilon \in \mathbb{R}$. If we define a function $\gamma(t), t \in(0 ; 1]$, in the following way:

$$
\gamma(t)= \begin{cases}\gamma_{n}, & \text { if } t=\frac{1}{n}, n \in \mathbb{N} \\ \text { monotonic, } & \text { if } \frac{1}{n+1}<t<\frac{1}{n}, n \in \mathbb{N}\end{cases}
$$

then the sequence $\left\{n^{\varepsilon} \gamma_{n}\right\}$ is almost decreasing (increasing) if and only if the function $t^{-\varepsilon} \gamma(t)$ is almost increasing (decreasing) on $(0,1)$, i.e. $t_{1}^{-\varepsilon} \gamma\left(t_{1}\right) \leq$ $K t_{2}^{-\varepsilon} \gamma\left(t_{2}\right) \quad(\geq)$ for $0<t_{1} \leq t_{2}<1$. 
Proof. It is clear that if $t^{-\varepsilon} \gamma(t)$ is an almost increasing function, then $\left\{n^{\varepsilon} \gamma_{n}\right\}$ is an almost decreasing sequence.

Let $\left\{n^{\varepsilon} \gamma_{n}\right\}$ be an almost decreasing function, i.e. there exists a constant $A \geq 1$ such that $l^{\varepsilon} \gamma_{l} \leq A s^{\varepsilon} \gamma_{s}$ for any $s \leq l$. Let $0<t_{1} \leq t_{2} \leq 1, \frac{1}{n+1}<t_{1} \leq \frac{1}{n}$ and $\frac{1}{m+1}<t_{2} \leq \frac{1}{m}(n, m \in \mathbb{N})$. If $n=m$, then we have $t_{1}^{-\varepsilon} \gamma\left(t_{1}\right) \leq L t_{2}^{-\varepsilon} \gamma\left(t_{2}\right)$ with $L:=A \cdot 4^{|\varepsilon|}$. Indeed, if $\gamma_{n+1} \leq \gamma_{n}$, then $\gamma\left(t_{1}\right) \leq \gamma\left(t_{2}\right)$ and $t_{1}^{-\varepsilon} \gamma\left(t_{1}\right) \leq L t_{2}^{-\varepsilon} \gamma\left(t_{2}\right)$. If $\gamma_{n} \leq \gamma_{n+1}$, then

$$
\frac{\gamma\left(t_{1}\right)}{t_{1}^{\varepsilon}} \leq 2^{|\varepsilon|} \gamma_{n+1}(n+1)^{\varepsilon} \leq A \cdot 2^{|\varepsilon|} \gamma_{n} n^{\varepsilon} \leq A \cdot 4^{|\varepsilon|} \frac{\gamma\left(t_{2}\right)}{t_{2}^{\varepsilon}}
$$

In fact, we have for $k \in \mathbb{N}$

$$
\frac{1}{L} \gamma_{k+1}(k+1)^{\varepsilon} \leq t^{-\varepsilon} \gamma(t) \leq L \gamma_{k} k^{\varepsilon} \quad \text { for any } t \in\left(\frac{1}{k+1} ; \frac{1}{k}\right]
$$

that implies for any $t_{1}, t_{2}\left(0<t_{1} \leq t_{2} \leq 1\right)$

$$
t_{1}^{-\varepsilon} \gamma\left(t_{1}\right) \leq L n^{\varepsilon} \gamma_{n} \leq A L(m+1)^{\varepsilon} \gamma_{m+1} \leq A L^{2} t_{2}^{-\varepsilon} \gamma\left(t_{2}\right)
$$

i.e. $t^{-\varepsilon} \gamma(t)$ is an almost increasing function on $(0,1)$. The proof for an almost increasing sequence is analogous.

Lemma 3.6. Let $\gamma=\left\{\gamma_{n}\right\}$ be a positive sequence and $\mu=\left\{\mu_{n}\right\}$ be a nonnegative sequence, and $\beta>0$.

(A) If $\gamma \in S Q$ and $\gamma \in S Q_{\beta}$, then the following conditions are equivalent:

$$
\begin{gathered}
\sum_{k=1}^{n} k^{\beta} \mu_{k}=O\left(\gamma_{n} n^{\beta}\right) \\
\sum_{k=n}^{\infty} \mu_{k}=O\left(\gamma_{n}\right)
\end{gathered}
$$

(B) Let $\gamma$ be a nonincreasing sequence. If for any sequence of nonnegative terms $\left\{\mu_{n}\right\}$ conditions (5) and (6) are equivalent, then $\gamma \in S Q$ and $\gamma \in$ $S Q_{\beta}$.

Proof. (A). Let $\gamma \in S Q$ and $\gamma \in S Q_{\beta}$. Then the sequence $\left\{n^{\beta} \gamma_{n}\right\}$ satisfies all conditions of Lemma 3.4 with $\delta=\beta$, and we have equivalence of (5) and (6).

(B). Let the conditions (5) and (6) be equivalent for any sequence of nonnegative terms $\left\{\mu_{n}\right\}$. We define $\left\{\mu_{n}:=\triangle \gamma_{n}\right\}_{n \in \mathbb{N}}$ where $\triangle \gamma_{n}=\gamma_{n}-\gamma_{n+1}$. It is 
clear that $\left\{\mu_{n}\right\}$ satisfies (6), therefore $\left\{\mu_{n}\right\}$ satisfies (5). Then

$$
\begin{aligned}
2^{-n \beta} \sum_{k=0}^{n} 2^{k \beta} \gamma_{2^{k}} & \leq C 2^{-n \beta} \sum_{k=1}^{2^{n}} k^{\beta-1} \gamma_{k} \\
& =2^{-n \beta} \sum_{k=1}^{2^{n}} k^{\beta-1}\left(\sum_{l=k}^{2^{n}} \triangle \gamma_{l}+\gamma_{2^{n}+1}\right) \\
& \leq 2^{-n \beta} \sum_{l=1}^{2^{n}} \triangle \gamma_{l}\left(\sum_{k=1}^{l} k^{\beta-1}\right)+O\left(\gamma_{2^{n}+1}\right) \\
& =O\left(2^{-n \beta} \sum_{l=1}^{2^{n}} l^{\beta} \triangle \gamma_{l}+\gamma_{2^{n}}\right) \\
& =O\left(\gamma_{2^{n}}\right) .
\end{aligned}
$$

Then by Lemmas 3.1 and 3.3 we have that the sequence $\left\{2^{n \beta} \gamma_{2^{n}}\right\}$ is quasi geometrically increasing, and there exists $\varepsilon \in(0, \beta)$ such that the sequence $\left\{n^{\beta-\varepsilon} \gamma_{n}\right\}$ is almost increasing, i.e. $\gamma \in S Q_{\beta}$. Therefore,

$$
\sum_{k=1}^{n} k^{\beta-1} \gamma_{k}=\sum_{k=1}^{n} k^{\beta-\varepsilon} \gamma_{k} k^{\varepsilon-1}=O\left(n^{\beta-\varepsilon} \gamma_{n} \sum_{k=1}^{n} k^{\varepsilon-1}\right)=O\left(n^{\beta} \gamma_{n}\right)
$$

i.e. the sequence $\left\{y_{n}=\frac{1}{n} \gamma_{n}\right\}$ satisfies (5) as well as (6). Then

$$
\begin{aligned}
\sum_{k=n}^{\infty} \gamma_{2^{k}} & \leq C \sum_{k=n}^{\infty} \gamma_{2^{k}} \sum_{\xi=2^{k}+1}^{2^{k+1}} \xi^{-1} \\
& \leq C \sum_{k=2^{n}+1}^{\infty} \gamma_{k} k^{-1} \\
& =O\left(\gamma_{2^{n}}\right)
\end{aligned}
$$

and by Lemmas 3.1 and 3.3 we have $\gamma \in S Q$.

Lemma 3.7. ([3, p. 72]). For positive $f(x)$ on $(0, X], X>0$ we have

$$
\begin{aligned}
& m(f)=\sup \left\{\alpha \in \mathbb{R}: x^{-\alpha} f(x) \text { is almost increasing }\right\} \\
& M(f)=\inf \left\{\beta \in \mathbb{R}: x^{-\beta} f(x) \text { is almost decreasing }\right\} .
\end{aligned}
$$

Lemma 3.8. ([1]). Let $f(x) \in C^{+}$and $f(x)=\sum_{n=1}^{\infty} a_{n} \cos n x, a_{n} \geq 0$. Then $\sum_{k=2 n}^{\infty} a_{k} \leq 4 E_{n}(f)$. 
Lemma 3.9. ([16]). Let $\beta>0$. If $f(x) \in C$, then $E_{n-1}(f) \leq C(\beta) \omega_{\beta}\left(f, \frac{1}{n}\right)$, $n \in \mathbb{N}$.

Lemma 3.10. ([15]). If $f(x) \in C$ such that $\sum_{n=1}^{\infty} n^{-1} E_{n}(f)<\infty$, then the conjugate function $\tilde{f}(x)$ is continuous and $E_{n}(\tilde{f}) \leq C\left(E_{n}(f)+\sum_{k=n+1}^{\infty} k^{-1} E_{k}(f)\right)$, $n \in \mathbb{N}$.

\section{Results on majorants}

N.K. Bari and S.B. Stechkin proved ${ }^{4}$ two following lemmas.

Lemma 4.1. ([2]) Let $\varphi(\cdot) \in \Phi$. Then the following conditions are equivalent:

(B) $\quad \sum_{k=n+1}^{\infty} \frac{1}{k} \varphi\left(\frac{1}{k}\right)=O\left[\varphi\left(\frac{1}{n}\right)\right]$

(S) There exists a constant $\alpha(0<\alpha<1)$ such that the function $t^{-\alpha} \varphi(t)$ is almost increasing in the sense of Bernstein ${ }^{5}$, that is $t_{1}^{-\alpha} \varphi\left(t_{1}\right) \leq C t_{2}^{-\alpha} \varphi\left(t_{2}\right)$ $\left(t_{1} \leq t_{2}\right)$.

Lemma 4.2. ([2]) Let $\varphi(\cdot) \in \Phi$. Then the following conditions are equivalent:

$\left(B_{\beta}\right) \sum_{k=1}^{n} k^{\beta-1} \varphi\left(\frac{1}{k}\right)=O\left[n^{\beta} \varphi\left(\frac{1}{n}\right)\right]$

$\left(S_{\beta}\right)$ There exists a constant $\alpha(0<\alpha<\beta)$ such that the function $t^{\alpha-\beta} \varphi(t)$ is almost decreasing in the sense of Bernstein, that is $t_{2}^{\alpha-\beta} \varphi\left(t_{2}\right) \leq C t_{1}^{\alpha-\beta} \varphi\left(t_{1}\right)$ $\left(t_{1} \leq t_{2}\right)$.

We shall obtain the conditions that are equivalent to the conditions of the first and the second group. First, for the sequence $\gamma:=\left\{\gamma_{n}\right\}$ of positive numbers let us define the following conditions of lacunarity ${ }^{6}$ :

$\left(\Lambda^{1}\right)$ The sequence $\gamma$ satisfies the condition $\sum_{k=1}^{n} \gamma_{k} \leq C \gamma_{n}(n \in \mathbb{N}, C \geq 1)$.

$\left(\Lambda^{2}\right)$ There exist natural numbers $\mu:=\mu(\gamma)$ and $N:=N(\gamma)$ such that the inequality $\gamma_{n+\mu} \geq 2 \gamma_{n}$ holds for any natural $n \geq N$.

$\left(\Lambda^{3}\right)$ The sequence $\gamma$ can be presented as a union of finite number of sequences $\mathrm{N}$ which are lacunary starting from some member, i.e., $\gamma \in \bigcup_{s=1}^{r} \mathrm{~N}^{(s)}$.

$\left(\bar{\Lambda}^{1}\right)$ The sequence $\gamma$ satisfies the condition $\sum_{k=n}^{\infty} \gamma_{k} \leq C \gamma_{n}(n \in \mathbb{N}, C \geq 1)$.

$\left(\bar{\Lambda}^{2}\right)$ There exist natural numbers $\mu:=\mu(\gamma)$ and $N:=N(\gamma)$ such that the inequality $\gamma_{n+\mu} \leq \frac{1}{2} \gamma_{n}$ holds for any natural $n \geq N$.

\footnotetext{
${ }^{4}$ Here we recall only a part of their results, which we shall use further.

${ }^{5}$ See $[2]$.

${ }^{6}$ Note that the similar conditions were considered by L. Leindler [8], S.B. Stechkin [14] and V. Totik [17].
} 
$\left(\bar{\Lambda}^{3}\right)$ The sequence $\kappa=\left\{\frac{1}{\gamma_{n}}\right\}$ can be presented as a union of finite number of sequences $\mathrm{N}$ which are lacunary starting from some member, i.e., $\kappa \in$ $\bigcup_{s=1}^{r} \mathrm{~N}^{(s)}$.

Lemma 4.3. Let $\gamma=\left\{\gamma_{n}\right\}$ be the sequence of positive numbers. Then the following statements are true:

(a). $\gamma \in \Lambda^{1} \Longrightarrow \gamma \in \Lambda^{2} \Longrightarrow \gamma \in \Lambda^{3}$

(b). $\gamma \in \Lambda^{1} \Longleftrightarrow \gamma \in \Lambda^{2} \cap L A I \Longleftrightarrow \gamma \in \Lambda^{3} \cap L A I$

(c). $\gamma \in \bar{\Lambda}^{1} \Longrightarrow \gamma \in \bar{\Lambda}^{2} \Longrightarrow \gamma \in \bar{\Lambda}^{3}$

(d). $\gamma \in \bar{\Lambda}^{1} \Longleftrightarrow \gamma \in \bar{\Lambda}^{2} \cap L A D \Longleftrightarrow \gamma \in \bar{\Lambda}^{3} \cap L A D$.

Proof. (a). Let $\gamma \in \Lambda_{1}$. Then by Lemma 3.1 it is quasi geometrically increasing and so $\gamma \in \Lambda_{2}$, i.e. there exist natural numbers $\mu:=\mu(\gamma)$ and $K:=K(\gamma)$ such that the inequality $\frac{\gamma_{k+\mu}}{\gamma_{k}} \geq 2$ holds for any $k \geq K$. Following [14], we define the following subsequences of $\gamma: \gamma_{k}^{(s)}=\gamma_{(k-1) \mu+s}, s=1, \cdots, \mu$. Then

$$
\frac{\gamma_{k+1}^{(s)}}{\gamma_{k}^{(s)}} \geq 2
$$

holds for $k$ starting with some number $K_{s}, s=1, \cdots, \mu$, i.e., $\left\{\gamma_{k}^{(s)}\right\} \in \mathrm{N}^{(s)}, s=$ $1, \cdots, \mu$ and $\gamma \in \bigcup_{s=1}^{\mu} \mathrm{N}^{(s)}$.

(b). By Lemma 3.1, we have $\gamma \in \Lambda_{1} \Longrightarrow \gamma \in L A I$. Then it is sufficient to prove that if $\gamma \in L A I$, then $\gamma \in \Lambda_{3} \Longrightarrow \gamma \in \Lambda_{1}$. Let $\gamma \in \bigcup_{s=1}^{r} \mathrm{~N}^{(s)}$, i.e., there exist natural numbers $K_{s}$, such that the inequalities

$$
\frac{\gamma_{k+1}^{(s)}}{\gamma_{k}^{(s)}} \geq \lambda_{s}>1, \quad s=1, \cdots, r
$$

hold for any $k \geq K_{s}$. Put

$$
\begin{aligned}
K & :=\max \left\{l \in \mathbb{N}: \gamma_{l}=\gamma_{K_{s}}^{(s)}, s=1, \cdots, r\right\} \\
C^{*} & :=\max _{s=1, \cdots, K}\left(\gamma_{s}^{-1} \sum_{t=1}^{s} \gamma_{t}\right) .
\end{aligned}
$$

Then for any $M \leq K$ the following is true $\sum_{l=1}^{M} \gamma_{l} \leq C^{*} \gamma_{M}$. Further, let us consider a fixed integer $M>K$. It is clear, that each member of the sequence $\left\{\gamma_{t}\right\}_{t=K}^{M}$ belongs to some sequence $\mathbf{N}^{(s)}$, i.e.

$$
\left\{\gamma_{K}, \cdots, \gamma_{M}\right\} \subset \bigcup_{i=1}^{d} \mathrm{~N}^{\left(s_{i}\right)} \quad 1 \leq d \leq r .
$$


Define

$$
M_{s_{i}}:=\max \left\{l \in \mathbb{N}: \gamma_{l}^{\left(s_{i}\right)} \in\left\{\gamma_{K}, \cdots, \gamma_{M}\right\}\right\}, \quad i=1, \cdots, d
$$

Then $(i=1, \cdots, d)$

$$
\sum_{l=K_{s_{i}}}^{M_{s_{i}}} \gamma_{l}^{\left(s_{i}\right)}=\gamma_{M_{s_{i}}}^{\left(s_{i}\right)} \sum_{l=K_{s_{i}}}^{M_{s_{i}}} \frac{\gamma_{l}^{\left(s_{i}\right)}}{\gamma_{M_{s_{i}}}^{\left(s_{i}\right)}} \leq \gamma_{M_{s_{i}}}^{\left(s_{i}\right)} \sum_{l=K_{s_{i}}}^{M_{s_{i}}} \lambda_{s_{i}}^{l-M_{s_{i}}}=C \gamma_{M_{s_{i}}}^{\left(s_{i}\right)}
$$

and therefore

$$
\sum_{l=K}^{M} \gamma_{l} \leq \sum_{i=1}^{d} \sum_{l=K_{s_{i}}}^{M_{s_{i}}} \gamma_{l}^{\left(s_{i}\right)} \leq C_{1} \sum_{i=1}^{d} \gamma_{M_{s_{i}}}^{\left(s_{i}\right)}=: C_{1} A
$$

Without loss of generality, let us denote by $s_{1}$ the number of the sequence such that $\gamma_{M_{s_{1}}}^{\left(s_{1}\right)}=\gamma_{M}$. If $d=1$, then $A=\gamma_{M}$; if $d=2$, then we shall use the condition $\gamma \in L A I$ and the inequality (8) to get

$$
A=\gamma_{M}+\gamma_{M_{s_{2}}}^{\left(s_{2}\right)} \leq C\left(\gamma_{M}+\sum_{l=K_{s_{1}}}^{M_{s_{1}}} \gamma_{l}^{\left(s_{1}\right)}\right) \leq C \gamma_{M}
$$

Similarly, if $d>2$, then

$$
A=\gamma_{M}+\sum_{i=2}^{d} \gamma_{M_{s_{i}}}^{\left(s_{i}\right)} \leq C\left(\gamma_{M}+\sum^{\prime}\right)
$$

where $\sum^{\prime}$ contains $d-2$ terms. Repeating the reasoning, we shall get $A \leq C \gamma_{M}$. In such a way, for any $M>K$ we have the inequality

$$
\sum_{l=1}^{M} \gamma_{l} \leq\left(C^{*}+1\right) \sum_{l=K}^{M} \gamma_{l} \leq C \gamma_{M}
$$

i.e., $\gamma \in \Lambda_{1}$.

Statements (c) and (d) follow from (a) and (b). Indeed, if we define the sequence $\frac{1}{\gamma}:=\left\{\frac{1}{\gamma_{n}}\right\}$, we obtain that $\gamma \in \Lambda_{1} \Longleftrightarrow \frac{1}{\gamma} \in \bar{\Lambda}^{1}$ (this follows from Lemma 3.1), $\gamma \in \Lambda_{2} \Longleftrightarrow \frac{1}{\gamma} \in \bar{\Lambda}^{2}$ (trivial), $\gamma \in \Lambda_{3} \Longleftrightarrow \frac{1}{\gamma} \in \bar{\Lambda}^{3}$ (trivial).

Remark 4.4. There are examples of sequences $\gamma_{1}$ and $\gamma_{2}$, which are not local almost increasing and such that $\gamma_{1} \in \Lambda^{2}$ but $\gamma_{1} \notin \Lambda^{1}$ and $\gamma_{2} \in \Lambda^{3}$ but $\gamma_{2} \notin \Lambda^{2}$. There exist similar counter examples for the item (c) of sequences $\gamma_{3}$ and $\gamma_{4}$, which are not local almost decreasing. 
Proof. Let us consider $\gamma=\left(\gamma^{(1)}, \gamma^{(2)}\right)$, where $\gamma^{(1)}=\left\{\gamma_{l}^{(1)}=2^{l}\right\}$ and $\gamma^{(2)}=$ $\left\{\gamma_{l}^{(2)}=2^{2^{l}}\right\}$. If the members of the sequence $\gamma$ are placed in the following way: for $n=2 m: \gamma_{n}=\gamma_{n}^{(1)}$, and for $n=2 m+1: \gamma_{n}=\gamma_{n}^{(2)}$, then it is clear that $\gamma \in \Lambda_{2}$ but $\gamma \notin \Lambda_{1}$. On the other hand, let the elements of the sequence $\gamma$ be placed in the following order: if $n=2^{s}$, then $\left\{\gamma_{n}=\gamma_{n}^{(1)}\right\}$, and if $n \neq 2^{s}$, then $\left\{\gamma_{n}=\gamma_{n}^{(2)}\right\}$. Then $\gamma \in \Lambda_{3}$, but $\gamma \notin \Lambda_{2}$.

Now we provide the interrelation between several conditions on positive functions. In particular, we study conditions on majorants of moduli of smoothness from Theorems $1.1-1.5$.

Lemma 4.5. Let $\varphi(\cdot) \in \Phi$. Then the following conditions are equivalent:

(B) $\quad \sum_{k=n+1}^{\infty} \frac{1}{k} \varphi\left(\frac{1}{k}\right)=O\left[\varphi\left(\frac{1}{n}\right)\right]$

$(\bar{\Lambda}) \quad$ The sequence $\delta=\left\{\delta_{\nu}=\varphi\left(\frac{1}{2^{\nu}}\right)\right\}_{\nu \in \mathbb{N}}$ satisfies any of the conditions $\left(\bar{\Lambda}^{i}\right)$ for $i=1,2,3$.

(SQ) There exists a constant $\alpha \in(0,1)$ such that the sequence $\left\{n^{\alpha} \varphi\left(\frac{1}{n}\right)\right\}_{n \in \mathbb{N}}$ is almost decreasing.

$(M) \quad m(\varphi)>0$

$\left(\bar{\Omega}^{(2)}\right) \varphi \in \bigcup_{\alpha>0} \Omega_{\alpha}^{(2)}$.

Proof. Step 1: $\varphi \in B \Leftrightarrow \delta \in \bar{\Lambda}$, where $\delta:=\left\{\delta_{\nu}=\varphi\left(\frac{1}{2^{\nu}}\right)\right\}_{\nu \in \mathbb{N}}$. Due to the monotonicity of $\varphi$ we have $\varphi \in B \Leftrightarrow \sum_{\nu=n}^{\infty} \varphi\left(\frac{1}{2^{\nu}}\right)=O\left[\varphi\left(\frac{1}{2^{n}}\right)\right]$ and by Lemma 4.3 we have $\varphi \in B \Leftrightarrow \delta \in \bar{\Lambda}^{i}$ for any $i=1,2,3$.

Step 2: $\varphi \in B \Leftrightarrow \varphi \in S Q$. Indeed, if $\varphi \in \Phi$, then $\varphi \in B \Leftrightarrow \varphi \in S$ by Lemma 4.1 , and we write $\varphi \in S \Leftrightarrow \varphi \in S Q$ by Lemma 3.5.

Step 3: $\varphi \in B \Leftrightarrow \varphi \in M$. By Lemma 4.1, if $\varphi \in B$, then there exists $\varepsilon>0$ such that $t^{-\varepsilon} \varphi(t)$ is almost increasing function on $(0,1)$. Then Lemma 3.7 implies $m(\varphi) \geq \varepsilon>0$, i.e., $\varphi \in M$. Conversely, if $\varphi \in M$, then from Lemma 3.7 we have that for any $\varepsilon \in(0, m(\varphi))$ the function $t^{-\varepsilon} \varphi(t)$ is almost increasing and, by Lemma 4.1, $\varphi \in B$.

Step 4: $\varphi \in B \Rightarrow \varphi \in \bar{\Omega}^{(2)} \equiv \bigcup_{\alpha>0} \Omega_{\alpha}^{(2)}$. Let $\varphi \in B$, then $\varphi \in S Q$, i.e., there exists a constant $\alpha \in(0,1)$ such that $l^{\alpha} \varphi\left(\frac{1}{l}\right) \leq A s^{\alpha} \varphi\left(\frac{1}{s}\right)$ holds for any $s \leq l$. Put $s=2^{n}, l=2^{n+\mu}$, then for any $n, \mu$ it holds $2^{\mu \alpha} \varphi\left(\frac{1}{2^{n+\mu}}\right) \leq A \varphi\left(\frac{1}{2^{n}}\right)$.

If $A \leq 2$, then $\varphi \in \Omega_{\alpha}^{(2)} \subset \bigcup_{\alpha>0} \Omega_{\alpha}^{(2)}$.

Let $A>2$. Put $\mu^{\prime}=\min \left\{\mu \in \mathbb{N}: A \leq 2^{\mu \alpha}\right\}$. It is clear that $\mu^{\prime} \alpha>1$, and if we define $\alpha^{\prime}:=1 / \mu^{\prime}$, then we shall have $A \leq 2^{\mu^{\prime} \alpha} \leq 2^{1+\mu\left(\alpha-\alpha^{\prime}\right)}$ for all integers $\mu \geq \mu^{\prime}$ and $2^{\mu \alpha^{\prime}} \leq 2$ for all integer $\mu \leq \mu^{\prime}$. Then the following two conditions hold: 
(*) For any integer $\mu \geq \mu^{\prime}$ the following inequality is true:

$$
2^{\mu \alpha^{\prime}} \varphi\left(\frac{1}{2^{n+\mu}}\right) \leq \frac{2}{A} 2^{\mu\left(\alpha-\alpha^{\prime}\right)} 2^{\mu \alpha^{\prime}} \varphi\left(\frac{1}{2^{n+\mu}}\right) \leq 2 \varphi\left(\frac{1}{2^{n}}\right), \quad n \in \mathbb{N} .
$$

(**) For any integer $\mu \leq \mu^{\prime}$ the following inequality is true:

$$
2^{\mu \alpha^{\prime}} \varphi\left(\frac{1}{2^{n+\mu}}\right) \leq 2 \varphi\left(\frac{1}{2^{n+\mu}}\right) \leq 2 \varphi\left(\frac{1}{2^{n}}\right), \quad n \in \mathbb{N}
$$

Then, $\varphi \in \Omega_{\alpha^{\prime}}^{(2)} \subset \bigcup_{\alpha>0} \Omega_{\alpha}^{(2)}$.

Step 5: $\varphi \in \bar{\Omega}^{(2)} \Rightarrow \varphi \in B$. Let there exist a $\kappa>0$ such that $\varphi \in \Omega_{\kappa}^{(2)}$, i.e., for every natural $\mu$ there exists a natural number $N:=N(\mu)$ such that

$$
2^{\mu \kappa} \varphi\left(\frac{1}{2^{n+\mu}}\right) \leq 2 \varphi\left(\frac{1}{2^{n}}\right) \text { for all } n>N
$$

Then there exists an integer $\mu=\mu(\kappa)$ such that the inequality

$$
\varphi\left(\frac{1}{2^{n+\mu}}\right) \leq \frac{1}{2} \varphi\left(\frac{1}{2^{n}}\right) \text { for all } n>N
$$

holds. Therefore, the sequence $\delta=\left\{\delta_{\nu}=\varphi\left(\frac{1}{2^{\nu}}\right)\right\}_{\nu \in \mathbb{N}}$ satisfies the $\bar{\Lambda}^{2}$ condition, that implies $\varphi \in B$ (as we have already proved).

Remark 4.6. For any $\kappa>0$ we have $\Omega_{\kappa}^{(2)} \cap \Phi \subset B \cap \Phi \subset \Omega_{0}^{(2)} \cap \Phi$.

Proof. By Lemma $4.5 \varphi \in B \Leftrightarrow \varphi \in \bigcup_{\alpha>0} \Omega_{\alpha}^{(2)}$, and for any $\kappa>0$ we have $\Omega_{\kappa}^{(2)} \subset B$. On the other hand, $B \subset \Omega_{0}^{(2)}$ since for any integer $\mu$ there exists an integer $N=N(\mu)$ such that

$$
\varphi\left(\frac{1}{2^{n+\mu}}\right) \leq 2^{\mu \kappa} \varphi\left(\frac{1}{2^{n+\mu}}\right) \leq 2 \varphi\left(\frac{1}{2^{n}}\right) \text { for any } n>N .
$$

We write the examples: $\varphi_{1}(\delta)=\delta^{\frac{\kappa}{2}}\left(\in B\right.$ but $\left.\notin \Omega_{\kappa}^{(2)}\right)$ and $\varphi_{2}(\delta)=\left(\ln \frac{1}{\delta}\right)^{-1}$ $\left(\in \Omega_{0}^{(2)}\right.$ but $\left.\notin B\right)$.

Lemma 4.7. Let $\varphi(\cdot) \in \Phi$. Then the following conditions are equivalent:

$\left(B_{\beta}\right) \quad \sum_{k=1}^{n} k^{\beta-1} \varphi\left(\frac{1}{k}\right)=O\left[n^{\beta} \varphi\left(\frac{1}{n}\right)\right]$

$\left(\Lambda_{\beta}\right) \quad$ The sequence $\delta=\left\{\delta_{\nu}=2^{\nu \beta} \varphi\left(\frac{1}{2^{\nu}}\right)\right\}_{\nu \in \mathbb{N}}$ satisfies any of the conditions $\left(\Lambda^{i}\right)$ for $i=1,2$.

$\left(S Q_{\beta}\right)$ There exists a constant $\alpha \in(0, \beta)$ such that the sequence $\left\{n^{\beta-\alpha} \varphi\left(\frac{1}{n}\right)\right\}_{n \in \mathbb{N}}$ is almost increasing. 
$\left(M_{\beta}\right) \quad M(\varphi)<\beta$

$\left(\bar{\Omega}_{\beta}^{(1)}\right) \varphi \in \bigcup_{0<\beta^{\prime}<\beta} \Omega_{\beta^{\prime}}^{(1)}$.

Proof. Step 1: $\varphi \in B_{\beta} \Leftrightarrow \delta \in \Lambda_{\beta}$, where $\delta:=\left\{\delta_{\nu}=2^{\nu \beta} \varphi\left(\frac{1}{2^{\nu}}\right)\right\}_{\nu \in \mathbb{N}}$. First, by $\varphi(\cdot) \in \Phi$ and by Lemma 4.3 , we have $\varphi \in B_{\beta} \Leftrightarrow \sum_{\nu=1}^{n} 2^{\beta \nu} \varphi\left(\frac{1}{2^{\nu}}\right)=$ $O\left[2^{\beta n} \varphi\left(\frac{1}{2^{n}}\right)\right] \Leftrightarrow \delta \in \Lambda^{1} \Rightarrow \delta \in \Lambda^{2}$. Now let $\delta \in \Lambda^{2}$. Then, by monotonicity of $\varphi, \delta$ is local almost increasing and Lemma 4.3 implies $\varphi \in B_{\beta}$.

Step 2: $\varphi \in B_{\beta} \Leftrightarrow \varphi \in S Q_{\beta}$. This follows from The Lemmas 4.2 and 3.5.

Step 3: $\varphi \in B_{\beta} \Leftrightarrow \varphi \in M_{\beta}$. The proof of this fact is similar to the proof of $\varphi \in B \Leftrightarrow \varphi \in M$ in Lemma 4.5.

Step 4: $\varphi \in B_{\beta} \Leftrightarrow \varphi \in \bar{\Omega}_{\beta}^{(1)}$. We divide the proof into the following parts:

a) If $\beta^{\prime}<\beta$, then $\Omega_{\beta^{\prime}}^{(1)} \subset \Omega_{\beta}^{(1)}$.

b) If $\beta^{\prime}<\beta$, then $B_{\beta^{\prime}} \subset B_{\beta}$.

c) $B_{\beta} \subset \Omega_{\beta}^{(1)}$

d) $\bigcup_{0<\beta^{\prime}<\beta} \Omega_{\beta^{\prime}}^{(1)} \subset B_{\beta}$

e) $B_{\beta} \equiv \bigcup_{0<\beta^{\prime}<\beta} B_{\beta^{\prime}}^{(1)}$

f) $\bigcup_{0<\beta^{\prime}<\beta} B_{\beta^{\prime}}^{(1)} \subset \bigcup_{0<\beta^{\prime}<\beta} \Omega_{\beta^{\prime}}^{(1)}$

g) $B_{\beta} \subset \bigcup_{0<\beta^{\prime}<\beta} \Omega_{\beta^{\prime}}^{(1)}$.

Now we shall prove a) $-\mathrm{g}$ ).

a): It is evident from the definition of $\Omega_{\beta}^{(1)}$.

b) : It results from $S Q_{\beta^{\prime}} \subset S Q_{\beta}$ for $\beta^{\prime}<\beta$.

c) : Let $\varphi \in B_{\beta}$. Then there exist $0<\alpha<1$ and $C \geq 1$ such that $\varphi\left(\frac{1}{2^{n}}\right) \leq$ $C 2^{\nu(\beta-\alpha)} \varphi\left(\frac{1}{2^{n+\nu}}\right)(\nu, n \in \mathbb{N})$. Let us define $\xi:=\min \left\{\nu \in \mathbb{N}: 2^{\nu \alpha} \geq 2 C\right\}$. Then we have for all $\beta^{\prime}>\beta$

$$
2^{\beta^{\prime} \xi} \varphi\left(\frac{1}{2^{n+\xi}}\right)>2^{\beta \xi} \varphi\left(\frac{1}{2^{n+\xi}}\right) \geq 2 \varphi\left(\frac{1}{2^{n}}\right)
$$

holds for all $n$, i.e., $\varphi \in \Omega_{\beta}^{(1)}$.

d) : Let $\varphi \in \bigcup_{0<\alpha<\beta} \Omega_{\alpha}^{(1)}$. It follows from the fact that there exists an $\alpha(0<\alpha<\beta)$ such that $\varphi \in \Omega_{\alpha}^{(1)}$, i.e., for any $\alpha^{\prime}>\alpha$ there exists an integer $\mu=\mu\left(\alpha^{\prime}\right)$ such that

$$
2^{\alpha^{\prime} \mu} \varphi\left(\frac{1}{2^{n+\mu}}\right)>2 \varphi\left(\frac{1}{2^{\mu}}\right) \quad \forall n \in \mathbb{N} .
$$


Then

$$
2^{\alpha^{\prime}(\mu+n)} \varphi\left(\frac{1}{2^{n+\mu}}\right) \geq 2^{1+\alpha^{\prime} n} \varphi\left(\frac{1}{2^{n}}\right),
$$

and by definition $\delta=\left\{\delta_{\nu}=2^{\alpha^{\prime} \nu} \varphi\left(\frac{1}{2^{\nu}}\right)\right\}_{\nu \in \mathbb{N}} \in \Lambda^{2}$, and therefore $\varphi \in B_{\alpha^{\prime}}$. So, there exists $\alpha^{\prime}\left(0<\alpha^{\prime}<\beta\right)$ such that $\varphi \in B_{\alpha^{\prime}}$. By part b), $\varphi \in B_{\beta}$.

e): By part b), $\bigcup_{0<\beta^{\prime}<\beta} B_{\beta^{\prime}}^{(1)} \subset B_{\beta}$. On the other hand, if $\varphi \in B_{\beta}$, then $\varphi \in S Q_{\beta}$, i.e., there exists a constant $\alpha(0<\alpha<\beta)$ such that the sequence $\left\{n^{\beta-\alpha} \varphi\left(\frac{1}{n}\right)\right\}_{n \in \mathbb{N}}$ is almost increasing. Then the following condition is true: there exists an $\alpha(0<\alpha<\beta)$ such that for all $\alpha^{\prime}\left(0<\alpha^{\prime}<\alpha\right)$ there exists a $\kappa\left(0<\kappa<\beta-\alpha^{\prime}\right)$ such that the sequence $\left\{n^{\left(\beta-\alpha^{\prime}\right)-\kappa} \varphi\left(\frac{1}{n}\right)\right\}_{n \in \mathbb{N}}$ is almost increasing (as $\kappa$ we can take $\left.\alpha-\alpha^{\prime}\right)$. So, there exists an $\alpha(0<\alpha<\beta)$ such that for all $\alpha^{\prime}\left(0<\alpha^{\prime}<\alpha\right)$ we write $\varphi \in B_{\beta-\alpha^{\prime}}$ and, finally, $\varphi \in \bigcup_{\beta-\alpha<\beta^{\prime}<\beta} B_{\beta^{\prime}}^{(1)} \subset$ $\bigcup_{0<\beta^{\prime}<\beta} B_{\beta^{\prime}}^{(1)}$.

f) - g) : It is clear that part c) implies f) and parts e) and f) imply g). Therefore, we have $\varphi \in B_{\beta} \Longleftrightarrow \varphi \in \bigcup_{0<\alpha<\beta} \Omega_{\alpha}^{(1)}$ from d) and g).

Remark 4.8. For any $\kappa, \varepsilon>0$ we have $\Omega_{0}^{(1)} \cap \Phi \subset \Omega_{\kappa}^{(1)} \cap \Phi \subset B_{\kappa+\varepsilon} \cap \Phi$.

Proof. By definition, we can write $\Omega_{0}^{(1)} \cap \Phi \subset \Omega_{\kappa}^{(1)} \cap \Phi$. And by Lemma 4.7, $\Omega_{\kappa}^{(1)} \cap \Phi \subset B_{\kappa+\varepsilon} \cap \Phi$. On the other hand, there are the following examples: $\varphi_{2}(\delta)=\delta^{\kappa} \quad\left(\in \Omega_{\kappa}^{(1)}\right.$ but $\left.\notin \Omega_{0}^{(1)}\right)$ and $\varphi_{3}(\delta)=\delta^{\kappa+\frac{\varepsilon}{2}} \quad\left(\in B_{\kappa+\varepsilon}\right.$ but $\left.\notin \Omega_{\kappa}^{(1)}\right)$.

Remark 4.9. There exists $\varphi(\cdot) \in \Phi$ such that the sequence $\delta=\left\{\delta_{\nu}=2^{\nu \beta} \varphi\left(\frac{1}{2^{\nu}}\right)\right\}$ satisfies the $\left(\Lambda^{3}\right)$-condition but $\varphi(\cdot)$ does not satisfy $\left(B_{\beta}\right)$. However, for any $\varphi \in \Phi$ we have that $\varphi \in B_{\beta}$ implies $\delta \in \Lambda^{3}$.

Proof. Fix $\gamma>0$. We can define

$$
\varphi\left(\frac{1}{2^{\nu}}\right)=\frac{1}{2^{\gamma 2^{k}}} \text { for } 2^{k-1} \leq \nu<2^{k}, k \in \mathbb{N} \quad \text { and } \quad \delta=\left\{\delta_{\nu}=2^{\beta \nu} \varphi\left(\frac{1}{2^{\nu}}\right)\right\}_{\nu \in \mathbb{N}}
$$

Then one can represent the sequence $\delta$ in the following way: $\delta=\left\{\delta^{(1)}, \delta^{(2)}, \delta^{(3)}\right\}$, where

$$
\begin{aligned}
& \delta^{(1)}=\left\{\delta_{\nu}, 2^{2 l-1}<\nu<2^{2 l}, l \in \mathbb{N}\right\} \\
& \delta^{(2)}=\left\{\delta_{\nu}, \nu=2^{l}, l \in \mathbb{N} \cup 0\right\} \\
& \delta^{(3)}=\left\{\delta_{\nu}, 2^{2 l}<\nu<2^{2 l+1}, l \in \mathbb{N}\right\} .
\end{aligned}
$$

If $\beta>3 \gamma$, it is clear that $\delta^{(j)} \in \mathrm{N}, j=1,2,3$, i.e., $\delta \in \bigcup_{s=1}^{3} \mathrm{~N}^{(s)}$, i.e., $\delta \in \Lambda^{3}$. On the other hand, we write

$$
\frac{\delta_{2^{k}}}{\delta_{2^{k}-1}}=\frac{2^{\beta 2^{k}} 2^{\gamma 2^{k}}}{2^{\gamma 2^{k+1}} 2^{\beta\left(2^{k}-1\right)}}=\frac{2^{\beta}}{2^{\gamma 2^{k}}} \longrightarrow 0 \quad \text { as } k \rightarrow \infty .
$$


Thus, the sequence $\delta$ is not local almost increasing and, by Lemma 4.3, we have $\varphi \notin B_{\beta}$. Finally, we can define the function $\varphi(\cdot)$ to be linear over $\left(\frac{1}{2^{n+1}}, \frac{1}{2^{n}}\right)$.

Corollary 4.10. If $\varphi(\cdot) \in \Phi$, then for any $s, \beta>0$ we have $\varphi \in B \Leftrightarrow \varphi^{s} \in B$, i.e.,

$$
\sum_{k=n+1}^{\infty} \frac{1}{k} \varphi\left(\frac{1}{k}\right)=O\left[\varphi\left(\frac{1}{n}\right)\right] \Leftrightarrow\left(\sum_{k=n+1}^{\infty} \frac{1}{k} \varphi^{s}\left(\frac{1}{k}\right)\right)^{\frac{1}{s}}=O\left[\varphi\left(\frac{1}{n}\right)\right],
$$

and $\varphi \in B_{\beta} \Leftrightarrow \varphi^{s} \in B_{s \beta}$, i.e.,

$$
\sum_{k=1}^{n} k^{\beta-1} \varphi\left(\frac{1}{k}\right)=O\left[n^{\beta} \varphi\left(\frac{1}{n}\right)\right] \Leftrightarrow\left(\sum_{k=1}^{n} k^{s \beta-1} \varphi^{s}\left(\frac{1}{k}\right)\right)^{\frac{1}{s}}=O\left[n^{\beta} \varphi\left(\frac{1}{n}\right)\right] .
$$

Proof. The proof follows from $\varphi \in B \Leftrightarrow \delta \in \bar{\Lambda}^{3} \Leftrightarrow \delta^{s} \in \bar{\Lambda}^{3} \Leftrightarrow \varphi^{s} \in$ $B\left(\right.$ for $\delta_{\nu}=\varphi\left(\frac{1}{2^{\nu}}\right)$ ) and $\varphi \in B_{\beta} \Leftrightarrow \delta \in \Lambda^{2} \Leftrightarrow \delta^{s} \in \Lambda^{2} \Leftrightarrow \varphi^{s} \in B_{s \beta}$ (for $\left.\delta_{\nu}=2^{\nu \beta} \varphi\left(\frac{1}{2^{\nu}}\right)\right)$.

\section{Results on a Boas-type problem}

Now we prove the theorem on belonging of a function to the generalized Lipschitz class.

Theorem 5.1. Let $\gamma=\left\{\gamma_{n}\right\}$ be the positive sequence and $\beta>0$. Then:

(A). If $\gamma \in S Q$ and $\gamma \in S Q_{\beta}$, then for any function $f \in C^{+}$with Fourier series (2) the conditions

$$
\begin{aligned}
\sum_{k=n}^{\infty}\left(\left|a_{k}\right|+\left|b_{k}\right|\right) & =O\left(\gamma_{n}\right) \\
\sum_{k=1}^{n} k^{\beta}\left(\left|a_{k}\right|+\left|b_{k}\right|\right) & =O\left(n^{\beta} \gamma_{n}\right) \\
\omega_{\beta}\left(f, \frac{1}{n}\right) & =O\left(\gamma_{n}\right)
\end{aligned}
$$

are equivalent.

(B). Let $\gamma$ be a non-increasing sequence. If for any function $f \in C^{+}$with Fourier series (2) the conditions (9), (10) and (11) are equivalent, then $\gamma \in S Q$ and $\gamma \in S Q_{\beta}$.

Proof. (A). Let $\gamma \in S Q$ and $\gamma \in S Q_{\beta}$. Then, by Lemma 3.6, the conditions (9) and (10) are equivalent. Now we shall prove that the conditions (9) and (11) are equivalent. 
Let function $f \in C^{+}$satisfy (9), and so, (10). Note, that if the $2 \pi$ periodic function $f(x)$ has a Fourier series $\sum_{n=-\infty}^{\infty} c_{n} e^{i n x}$, then $\triangle_{h}^{\beta} f\left(x-\frac{\beta h}{2}\right) \sim$ $\sum_{k \in \mathbb{Z}}\left(2 i \sin \frac{k h}{2}\right)^{\beta} c_{k} e^{i k x}$. For given $h$ one chooses $n$ according to $\frac{1}{n+1}<h \leq \frac{1}{n}$. Then

$$
\begin{aligned}
\left|\sum_{k \in \mathbb{Z}}\left(2 i \sin \frac{k h}{2}\right)^{\beta} c_{k} e^{i k x}\right| & \leq 2 \sum_{k=1}^{n}\left|2 \sin \frac{k h}{2}\right|^{\beta}\left|c_{k}\right|+2 \sum_{k=n+1}^{\infty}\left|2 \sin \frac{k h}{2}\right|^{\beta}\left|c_{k}\right| \\
& =J_{1}+J_{2} .
\end{aligned}
$$

Then, (10) and $\left|\sin \frac{k h}{2}\right| \leq \frac{k h}{2}$ imply $J_{1} \leq C h^{\beta} \sum_{k=1}^{n} k^{\beta}\left(\left|a_{k}\right|+\left|b_{k}\right|\right) \leq C \gamma_{n}$; and (9) implies $J_{2} \leq C \sum_{k=n+1}^{\infty}\left(\left|a_{k}\right|+\left|b_{k}\right|\right) \leq C \gamma_{n}$. Therefore, for any $f \in C^{+}$we have $\left|\triangle_{h}^{\beta} f\left(x-\frac{\beta h}{2}\right)\right| \leq C \gamma_{n}$ with $\frac{1}{n+1}<h \leq \frac{1}{n}$, and so $\omega_{\beta}\left(f, \frac{1}{n}\right)=O\left(\gamma_{n}\right)$. We have proved that (9) implies (11).

Further, we shall prove that (11) implies (9). Let $\xi \in C^{+}$have a Fourier series $\sum_{n=1}^{\infty}\left(a_{n}^{\prime} \cos n x+b_{n}^{\prime} \sin n x\right)$ and $\xi$ satisfy (11). Define the functions $\xi_{ \pm}(x)=\frac{\xi(x) \pm \xi(-x)}{2}$. Note, that $\omega_{\beta}\left(\xi_{ \pm}, \frac{1}{n}\right) \leq \omega_{\beta}\left(\xi, \frac{1}{n}\right)=O\left(\gamma_{n}\right)$. Using Lemma 3.8, Jackson's inequality (Lemma 3.9) and property of modulus of smoothness, we write

$$
\sum_{k=n}^{\infty}\left|a_{k}^{\prime}\right| \leq C E_{\left[\frac{n}{2}\right]}\left(\xi_{+}\right) \leq C(\beta) \omega_{\beta}\left(\xi_{+}, \frac{1}{n}\right)=O\left(\gamma_{n}\right) .
$$

Consider $\xi_{-}(x) \sim \sum_{n=1}^{\infty} b_{n}^{\prime} \sin n x$ and the conjugate function $\widetilde{\xi_{-}}$. The Fourier series of $\widetilde{\xi_{-}}$is $-\sum_{n=1}^{\infty} b_{n}^{\prime} \cos n x$, and in a similar manner as for (12) we shall use the Lemmas 3.8, 3.9, 3.10 and the condition $\gamma \in S Q$. Then

$$
\begin{aligned}
\sum_{k=n}^{\infty}\left|b_{k}^{\prime}\right| & \leq C E_{\left[\frac{n}{2}\right]}\left(\widetilde{\xi_{-}}\right) \\
& \leq C\left\{E_{\left[\frac{n}{2}\right]}\left(\xi_{-}\right)+\sum_{k=\left[\frac{n}{2}\right]+1}^{\infty} \frac{1}{k} E_{k}\left(\xi_{-}\right)\right\} \\
& \leq C(\beta)\left\{\omega_{\beta}\left(\xi_{-}, \frac{1}{n}\right)+\sum_{k=\left[\frac{n}{2}\right]+1}^{n} \frac{1}{k} \omega_{\beta}\left(\xi_{-}, \frac{1}{k}\right)+\sum_{k=n}^{\infty} \frac{1}{k} \omega_{\beta}\left(\xi_{-}, \frac{1}{k}\right)\right\} \\
& \leq C(\beta)\left\{\omega_{\beta}\left(\xi_{-}, \frac{1}{n}\right)+\sum_{k=n}^{\infty} \frac{1}{k} \omega_{\beta}\left(\xi_{-}, \frac{1}{k}\right)\right\} \\
& =O\left(\gamma_{n}+n^{\varepsilon} \gamma_{n} \sum_{k=n}^{\infty} k^{-\varepsilon-1}\right) \\
& =O\left(\gamma_{n}\right) .
\end{aligned}
$$


Thus,

$$
\sum_{k=n}^{\infty}\left(\left|a_{k}^{\prime}\right|+\left|b_{k}^{\prime}\right|\right)=O\left(\gamma_{n}\right),
$$

and (9) and part A of the theorem are true. The proof of part (B) follows from the equivalence of (9) and (10) by Lemma 3.6.

Remark 5.2. We see from the Lemmas 4.5 and 4.7 that part (A) of Theorem 5.1 for $\beta=1$ and $\beta=2$ implies all the theorems $1.1-1.5$.

Remark 5.3. In part (B) of Theorem 5.1, the condition that $\gamma$ is a nonincreasing sequence can be replaced by the condition $\gamma \in R_{0}^{+} B V S$.

Proof. It is enough to show part (B) of Lemma 3.6. Indeed, we consider the sequence $\left\{\mu_{n}=\left|\Delta \gamma_{n}\right|\right\}$ and repeat the reasoning of Lemma 3.6. To prove the inequality (7) we shall use the following fact: if the sequence $\gamma$ is $R^{+} B V S$, then $\gamma$ is almost decreasing.

Acknowledgement. This work was supported by the Russian Foundation for Fundamental Research (grant 03-01-00080) and the Leading Scientific Schools (grant NSH-1657.2003.1).

The author thanks Michele Bricchi, Elena Golovko and Mikhail K. Potapov for the attention to this work.

\section{References}

[1] Bari, N. K.: On best approximation of two conjugate functions by trigonometric polynomials. Izv. Akad. Nauk SSSR, Ser. Mat. 19 (1955)5, 285 - 302.

[2] Bari, N. K. and S. B. Stechkin: Best approximations and differential properties of two conjugate functions. Trudy Moskov. Mat. Obšč. 5 (1956), 483 - 522.

[3] Bingham, N. H., Goldie, C. M. and J. L. Teugels: Regular Variation. Cambridge: Cambridge Univ. Press 1987.

[4] Boas, R. P. Jr.: Fourier series with positive coefficients. J. Math. Anal. Appl. 17 (1967), $463-483$.

[5] Chan, L.- Y.: Generalized Lipschitz classes and asymptotic behavior of Fourier series. J. Math. Anal. Appl. 155 (1991)2, 371 - 377.

[6] Izumi, M. and S. Izumi: Lipschitz classes and Fourier coefficients. J. Math. Mech. 18 (1969), $857-870$.

[7] Leindler, L.: Strong approximation and generalized Lipschitz classes. In: Functional Analysis and Approximation (Proc. Conf. Oberwolfach 1980), ISNM 60. Basel: Birkhäuser-Verlag 1981, pp. 343 - 350.

[8] Leindler, L.: On the converses of inequalities of Hardy and Littlewood. Acta Sci. Math. 58 (1993)(1-4), 191 - 196. 
[9] Leindler, L.: Power-monotone sequences and Fourier series with positive coefficients. J. Inequal. Pure Appl. Math. 1 (2000)(1), Article 1. http://jipam.vu.edu.au/v1n1/001_99.html.

[10] Leindler, L.: A new class of numerical sequences and its applications to sine and cosine series. Analysis Math. 28 (2002), 279 - 286.

[11] Leindler, L. and J. Nemeth: On the connection between quasi power-monotone and quasi geometrical sequences with application to integrability theorems for power series. Acta Math. Hung. 68 (1995)(1-2), 7 - 19.

[12] Nemeth, J.: Fourier series with positive coefficients and generalized Lipschitz classes. Acta Sci. Math. (Szeged) 54(1990)(3-4 ), 291 - 304.

[13] Nemeth, J.: Power-monotone sequences and Fourier series with positive coefficients. J. Inequal. Pure Appl. Math. 2 (2001)(2), Article 14. http://jipam.vu.edu.au/v2n2/027_00.html

[14] Stechkin, S. B.: On absolute convergence of Fourier series. Izv. Akad. Nauk SSSR. Ser. Mat. 20 (1956), 385 - 412.

[15] Stechkin, S. B.: On best approximation of conjugate functions by trigonometric polynomials. Izv. Akad. Nauk SSSR, Ser. Mat. 20 (1956), 197 - 206.

[16] Taberski, R.: Differences, moduli and derivatives of fractional orders. Commentat. Math. 19 (1976-1977), 389 - 400.

[17] Totik, V.: On the strong approximation of Fourier series. Acta Math. Acad. Sci. Hung. 35 (1981), $151-172$.

Received 30.01.2004 